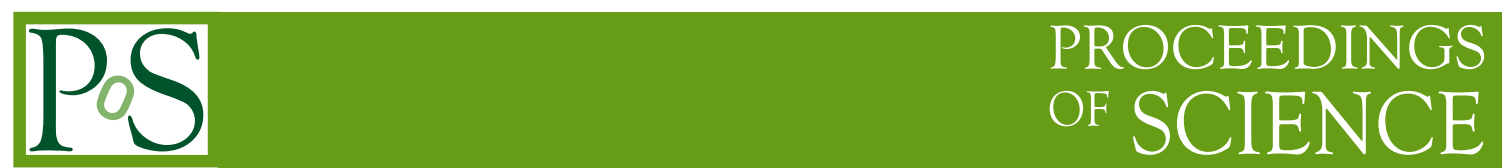

\title{
Interpretation of the top-quark mass results
}

\section{Gennaro Corcella*}

INFN, Laboratori Nazionali di Frascati

Via E. Fermi 40, 00044 Frascati (RM), Italy

E-mail: gennaro.corcella@lnf.infn.it

I discuss recent work aimed at interpreting the top-quark mass measurements at the LHC and determining the theoretical uncertainty.

XXV International Workshop on Deep-Inelastic Scattering and Related Subjects 3-7 April 2017

University of Birmingham, $U K$

${ }^{*}$ Speaker. 


\section{Introduction}

The top-quark mass is one of most important quantities of the Standard Model: it plays a role in the electroweak precision tests [1], since, together with the $W$ mass, it constrained the Higgs mass even before its discovery, as well as in the property of the Standard Model vacuum to lie on the border between stability and metastability regions [2]. Such results, however, rely on the assumption that the top-quark mass world average, i.e. $m_{t}=[173.34 \pm 0.27$ (stat) \pm 0.71 (syst) $] \mathrm{GeV}$ [3], corresponds to the pole mass and no further uncertainty associated with the interpretation of the measurements is accounted for.

In fact, the top-quark mass is determined by comparing experimental data with theory predictions: the extracted mass is the quantity $m_{t}$ in the calculation. In the following, I shall review the main methods used to reconstruct the top-quark mass at the LHC and discuss the ongoing work to interpret the top-mass results and determine the theoretical uncertainty. I will finally make some concluding remarks.

\section{Interpreting the top-quark mass measurements}

\subsection{Standard measurements}

Top-quark mass extraction strategies are typically labelled as standard or alternative measurements. The standard ones, based on the template, matrix-element and ideogram methods (see, e.g., the analyses in $[4,5])$, compare final-state distributions, associated with top-decay $(t \rightarrow b W)$ products, with Monte Carlo generators, such as HERWIG [6] or PYTHIA [7]. These programs simulate the hard scattering at leading order (LO), multiple radiation in the soft or collinear approximation and neglect the interference between top-production and decay phases. Most recent codes, such as aMC@NLO [8] and POWHEG [9], implement the hard scattering at next-to-leading order (NLO), but still rely on HERWIG and PYTHIA for showers and hadronization.

Much debate has been taking place through the years to connect the measured mass in standard methods, often called 'Monte Carlo' mass, with theoretical definitions like pole or $\overline{\mathrm{MS}}$ masses (see [10] for a review). On the one hand, unlike electrons, quarks do not exist as free particles and this is reflected in the fact that the pole mass exhibits the so-called renormalon ambiguity [11], associated with the infrared divergences in the higher-order corrections to the quark self energy. The induced uncertainty on the pole mass has been estimated to be roughly $\mathscr{O}\left(\Lambda_{\overline{\mathrm{MS}}}\right)$. Such an ambiguity has been lately reconsidered, in view of the recent calculation of the four-loop relation between pole and $\overline{\mathrm{MS}}$ masses, the latest being free from renormalons [12]. Refs. [13] and [14] computed the ultimate uncertainty on the top pole mass due to renormalons, obtaining about 110 and $250 \mathrm{MeV}$, respectively. Ref. [14] estimated the uncertainty by analyzing order by order the pole-mass series as a function of the $\overline{\mathrm{MS}}$ mass, while Ref. [13] compared the exact result in [12] with the asymptotic expansion in [11] and gauged the error by relying on a prescription on the computation of an inverse Borel transform. Though differing by more than a factor of 2 , nevertheless, both evaluations are smaller than the current uncertainty on $m_{t}$.

On the other hand, since the pole mass is defined in such a way to reabsorb all radiative corrections, the invariant mass of the products of the decay an on-shell top quark, as the top quark is treated in standard Monte Carlo codes, must be close to the pole mass. Nevertheless, much 
work has been carried out to determine the uncertainty on the measured mass and its identification with the top pole mass. One of the main contributions to the theoretical error is colour reconnection, namely the possibility that bottom quarks in top decays form a hadronic string (PYTHIA) or cluster (HERWIG) with (anti)quarks which do not belong to their own showers, but rather to initial-state radiation or $W$ decays. In the world-average analysis, colour reconnection accounts for about $300 \mathrm{MeV}$ on the total error on the top mass. Such a phenomenon clearly spoils the association of the mass of the top-decay products with the pole mass and has been investigated in [15] in terms of the PYTHIA string model and in [16] by using the HERWIG cluster model. In particular, Ref. [16] compared standard $t \bar{t}$ events with those yielded by the simulation of fictitious top-flavoured hadrons. In fact, if a $T$-hadron decays according to the spectator model, the $b$ quark is forced to form a cluster with either the spectator quark or the products of its own shower, while the colour connection with the initial state is suppressed. The investigation in [16], currently in progress, is also pretty interesting since, by applying lattice or Non Relativistic QCD, one is able to connect the mass of a top-flavoured hadron with any $m_{t}$ definition and it may thus serve as another benchmark analysis to assess the uncertainty on the Monte-Carlo-driven reconstructed top mass.

Furthermore, work has been undertaken to confront Monte Carlo distributions with resummed calculations performed in the framework of Soft Collinear Effective Theory (SCET), using the socalled MSR top mass definition, $m_{\mathrm{MSR}}(R)$, which, according to the value of $R$, may correspond, e.g., to the $\overline{\mathrm{MS}}$ or the pole mass. Ref. [17] expressed the pole mass in terms of the SCET jet mass in $e^{+} e^{-} \rightarrow t \bar{t}$ collisions, assuming that $m_{J}(\mu)$ should mimic the reconstructed $m_{t}$ for $\mu \simeq Q_{0}$, with $Q_{0}$ being the shower cutoff. A shift about $\delta m \sim \mathscr{O}\left(\alpha_{S} \Gamma\right) \sim 150-200 \mathrm{MeV}$, where $\Gamma$ is the top width, was then determined. More recently, Ref. [18] compared PYTHIA with a SCET calculation at NLO, including the resummation of next-to-next-to-leading soft- and collinear-enhanced logarithms (NNLL), for the 2-jettiness in $e^{+} e^{-} \rightarrow t \bar{t}$ processes, trying to calibrate the MSR mass in the resummation to reproduce the Monte Carlo spectrum. In fact, it was obtained that, within the error range, the mass parameter in PYTHIA is consistent with the tuned $m_{\mathrm{MSR}}(1 \mathrm{GeV})$, whereas it differs by about $(0.57 \pm 0.28) \mathrm{GeV}$ from the corresponding pole mass. The work in [18] was extended to $p p$ collisions in [19], where the extraction of $m_{t}$ from boosted top jets with light soft-drop grooming is proposed. By comparing a NLL resummation for the groomed top-jet mass with PYTHIA, the pole mass was found about 400-700 MeV below the calibrated PYTHIA mass. Another approach was suggested in [20]: one measures an observable, e.g. a total or differential $t \bar{t}$ cross section, without any assumption on the Monte Carlo parametrization, and, by comparing the data with the simulation, calibrates both observable and $m_{t}$. The conclusion of Ref. [20] is that, with the current precision on the inclusive $t \bar{t}$ cross section, the uncertainty on this calibration is roughly $2 \mathrm{GeV}$.

Sticking to Monte Carlo programs, a major improvement has been the recent release of the $b \bar{b} 4 \ell$ generator [21], which, within the POWHEG-BOX framework, simulates the full NLO process $p p \rightarrow b \bar{b} \ell^{+} v_{\ell} \ell^{-} \bar{v}_{\ell}$, including the interference between top production and decay, and nonresonant contributions. It will be therefore very interesting using this generator in template or matrix-element analyses and comparing the results with those yielded by standalone HERWIG or PYTHIA, as well as aMC@NLO or POWHEG with LO top decays. Furthermore, the feasibility of POWHEG to be interfaced with both HERWIG and PYTHIA should also shed light on the Monte Carlo systematics, due to the use of different parton showers and hadronization models. 


\subsection{Alternative measurements}

Other strategies to measure $m_{t}$, making use of total or differential cross sections, endpoints, energy peaks or kinematic properties of $t \bar{t}$ final states, are traditionally called 'alternative' measurements. The total $t \bar{t}$ cross section was calculated in the NNLO+NNLL approximation [22] and allows a direct determination of the pole mass. Both ATLAS and CMS Collaborations have measured the top mass from the inclusive cross section obtaining $m_{t}=\left(172.9_{-2.6}^{+2.5}\right) \mathrm{GeV}$ (ATLAS) [23] and $m_{t}=\left(173.6_{-1.8}^{+1.7}\right) \mathrm{GeV}$ (CMS) [24], combining 7 and $8 \mathrm{TeV}$ data. In principle, even this extraction depends on the Monte Carlo program used for the evaluation of the acceptance, but nonetheless the sensitivity to the implemented top mass turned out to be very mild. The errors in [23] and [24] are larger than those yielded by the standard methods; however, they are expected to decrease thanks to the higher statistics foreseen at the LHC Run II. The NNLO calculation of the $t \bar{t}$ cross section has been extended to differential distributions in [25] and the D0 Collaboration used it to measure the top-quark pole mass from the $t \bar{t}$ invariant mass or transverse momenta spectra, finding $m_{t}=(169.1 \pm 2.5) \mathrm{GeV}$ [26]. The top pole mass was also determined from the measurement of the $t \bar{t}+1$ jet cross section, which is more sensitive to $m_{t}$ than the inclusive $t \bar{t}$ rate, following Ref. [27], where the NLO $t \bar{t} j$ cross section was calculated through the POWHEG-BOX and matched to PYTHIA. The results are $m_{t}=\left(173.70_{-2.11}^{+2.28}\right) \mathrm{GeV}$ (ATLAS) [28] and $m_{t}=\left(169.90_{-3.66}^{+4.52}\right) \mathrm{GeV}$ (CMS) [29]; the impact of the Monte Carlo input mass in the evaluation of the acceptance is negligible. Lately, the NLO $t \bar{t}+1$ jet cross section has been calculated in terms of the $\overline{\mathrm{MS}}$ mass and compared with the LHC measurements [30]. The extracted value of the $\overline{\mathrm{MS}}$ mass is nevertheless consistent with the value which can be obtained from the pole mass.

Other proposed methods to reconstruct $m_{t}$ rely on kinematic properties of top-decay final states and hence, once again, the extracted mass must be close to the pole mass. Unlike the invariant mass of the top-decay products, used in the template analyses, quantities like energy peaks, endpoints or purely leptonic observables are however expected to exhibit a larger uncertainty due to higher-order corrections, which are not anymore reabsorbed in the mass definition.

In detail, it was found that the peak of the energy of the $b$-jet in top decay at LO is independent of the boost from the top to the laboratory frame, as well as of the production mechanism [31]. The CMS Collaboration measured the top mass from the $b$-jet energy peak and obtained $m_{t}=$ $[172.29 \pm 1.17$ (stat.) \pm 2.66 (syst.) $] \mathrm{GeV}$ at $8 \mathrm{TeV}$ [32].

The $b$-jet+lepton invariant-mass $\left(m_{b \ell}\right)$ spectrum was used by CMS to reconstruct $m_{t}$ in the dilepton channel: by comparing it with PYTHIA, $m_{t}=(172.3 \pm 1.3) \mathrm{GeV}$ was found [33]. The NLO calculation of $m_{b l}$ [34], performed in the narrow-width approximation with the pole mass, is also available and exhibits some disagreement with respect to LO parton showers [16, 35].

The endpoints of distributions like $m_{b \ell}, \mu_{b b}$ and $\mu_{\ell \ell}$, where $\mu_{b b}$ and $\mu_{\ell \ell}$ are related to the $b \bar{b}$ and $\ell^{+} \ell^{-}$invariant masses in the dilepton channel, were also explored to constrain $m_{t}$ [36]. Since $b$-flavoured jets can be calibrated directly from data, Monte Carlo uncertainties on the endpoints are mostly due to colour reconnection. The result, based on LO kinematics, is $m_{t}=$ $[173.9 \pm 0.9 \text { (stat. })_{-2.1}^{+1.7}$ (syst.) $] \mathrm{GeV}$. Updating the $B$-energy peak, $m_{b \ell}$ and endpoint analyses using novel data as well as the late NLO generator $b \bar{b} 4 \ell$ [21] is certainly worthwhile to be pursued for the sake of a more reliable estimate of the theoretical uncertainty.

Finally, purely leptonic observables in the dilepton channel, such as the Mellin moments of 
lepton energies or transverse momenta, were proposed to measure $m_{t}$ as they do not require the reconstruction of the top quark [37]. Such quantities do exhibit small hadronization effects, but they are sensitive to the production mechanism, to the Lorentz boost from the top rest frame to the laboratory frame, as well as to higher-order corrections. Preliminary analyses have yielded $m_{t}=$ $[171.70 \pm 1.10 \text { (stat. })_{-3.09}^{+2.68}$ (syst.) $] \mathrm{GeV}[38]\left(\mathrm{CMS}\right.$, based on LO MadGraph) and $m_{t}=(173.2 \pm$ 1.6) $\mathrm{GeV}$ [40] (ATLAS, based on the MCFM NLO code [39]) and are expected to be improved by matching NLO top-decay amplitudes with shower/hadronization generators.

\section{Conclusions}

I discussed the interpretation of the top mass measurements at the LHC: template-based determinations, relying on the reconstruction of the invariant mass of the top-decay products, yield results close to the top-quark pole mass, but nevertheless a careful determination of the theoretical uncertainty, of both perturbative and non-perturbative origin, such as missing higher orders, width corrections and colour-reconnection effects, is compelling. The late implementation of top decays and interference between production and decay phases at NLO should help to quantify the perturbative error on $m_{t}$. As for non-perturbative corrections, studies on colour reconnection or simulations of final states where the tops are forced to hadronize and the bottom quarks to form colour-singlet clusters with final-state partons will be useful to address the hadronization systematics.

As for top-mass definitions, two papers have addressed the renormalon ambiguity on the pole mass: although they disagree by roughly a factor of 2 , the estimated uncertainties are both below the current error on $m_{t}$. Studies aimed at relating the extracted mass to the pole mass have been carried out within the SCET formalism for $e^{+} e^{-}$processes and lately extended to $p p$ collisions. Alternative measurements, based on the comparison of the $t \bar{t}$ and $t \bar{t} j$ cross sections with NLO or NNLO calculations, allow a clean extraction of the pole or $\overline{\mathrm{MS}}$ mass, with errors which will decrease once the LHC statistics get higher. Other strategies, relying on kinematic properties of top-decay final states, have so far employed parton-shower generators with LO top decays: updates using NLO codes will lead to a more reliable estimate of the theoretical uncertainty.

In summary, given the latest LHC performances, top-quark phenomenology is on the road to become precision physics and the measurement of the top mass to reach a very high level of accuracy. In view of the implementation of advanced event generators for top physics, as well as of refined calculations for top production and decay, more accurate determinations of the top mass and, in particular, of the theoretical uncertainty are therefore both feasible and desirable.

\section{References}

[1] Gfitter Group, Eur. Phys. J. C74 (2014) 3046.

[2] G. Degrassi, S. Di Vita, J. Elias-Miró, J.R. Espinosa, G.F. Giudice, G. Isidori and A. Strumia, JHEP 1208 (2012) 098.

[3] ATLAS and CDF and CMS and D0 Collaborations, arXiv:1403.4427 [hep-ex].

[4] ATLAS Collaboration, Phys. Lett. B761 (2016) 350.

[5] CMS Collaboration, Phys. Rev. D93 (2016) 072004. 
[6] J. Bellm et al., Eur. Phys. J. C76 (2016) 196.

[7] T. Sjöstrand et al., Comput. Phys. Commun. 191 (2015) 159.

[8] J. Alwall et al., JHEP 1407 (2014) 079.

[9] S. Alioli, P. Nason, C. Oleari and E. Re, JHEP 1006 (2010) 043.

[10] G. Corcella, PoS TOP2015 (2016) 037.

[11] M. Beneke and V.N. Braun, Nucl. Phys. B426 (1994) 301.

[12] P. Marquard, A.V. Smirnov, V.A. Smirnov and M. Steinhauser, Phys. Rev. Lett. 114 (2015) 142002.

[13] M. Beneke, P. Marquard, P. Nason and M. Steinhauser, Phys. Lett. B775 (2017) 63.

[14] A.H. Hoang, C Lepenik and M. Preisser, JHEP 1709 (2017) 099.

[15] S. Argyropoulos and T. Sjöstrand, JHEP 1411 (2014) 043.

[16] G. Corcella, EPJ Web Conf. 80 (2014) 00019.

[17] S. Fleming, A.H. Hoang, S. Mantry, I.W. Stewart, Phys. Rev. D77 (2008) 114003.

[18] M. Butenschön, B. Denhadi, A.H. Hoang, V. Mateu, M. Preisser and I.W. Stewart, Phys. Rev. Lett. 117 (2016) 232001.

[19] A.H. Hoang, S. Mantry, A. Pathak and I.W. Stewart, arXiv:1708.02586 [hep-ph].

[20] J. Kieseler, K. Lipka and S.-O. Moch, Phys. Rev. Lett. 116 (2016) 122001.

[21] T. Jezo, J.M. Lindert, P. Nason, C. Oleari and S. Pozzorini, Eur. Phys. J. C76 (2016) 691.

[22] M. Czakon, P. Fiedler and A. Mitov, Phys. Rev. Lett. 110 (2013) 252004.

[23] ATLAS Collaboration, Eur. Phys. J. C74 (2015) 3109.

[24] CMS Collaboration, JHEP 08 (2016) 029.

[25] M. Czakon, D. Heymes and A. Mitov, Phys. Rev. Lett. 116 (2016) 082003.

[26] D0 Collaboration, D0 Note 6473-CONF.

[27] S. Alioli, P. Fernandez, J. Fuster, A. Irles, S.-O. Moch, P. Uwer and M. Vos, Eur. Phys. J. C73 (2013) 2438.

[28] ATLAS Collaboration, JHEP 1510 (2015) 121.

[29] CMS Collaboration, CMS-PAS-TOP-13-006.

[30] J. Fuster, D. Melini, P. Uwer and M. Vos, arXiv:1704.00540 [hep-ph].

[31] K. Agashe, R. Franceschini, D. Kim and M. Schulze, Eur. Phys. J. C76 (2016) 636.

[32] CMS Collaboration, PoS ICHEP2016 (2016) 743.

[33] CMS Collaboration, CMS-PAS-TOP-14-014.

[34] S. Biswas, K. Melnikov and M. Schulze, JHEP 1008 (2010) 048.

[35] G. Corcella and F. Mescia, Eur. Phys. J. C65 (2010) 171; Erratum-ibid. C68 (2010) 687.

[36] CMS Collaboration, Eur. Phys. J. C73 (2013) 2494.

[37] S. Frixione and A. Mitov, JHEP 1409 (2014) 012.

[38] CMS Collaboration, CMS-PAS-TOP-16-002.

[39] J.M. Campbell and R.K. Ellis, J. Phys. G42 (2015) 015005.

[40] ATLAS Collaboration, ATLAS-CONF-2017-044. 